\title{
A 3D shock-capturing model for free surface flow
}

\author{
G. Cannata, F. Lasaponara, F. Camilli, C. Petrelli \& F. Gallerano \\ Department of Civil, Construction and Environmental Engineering, \\ Sapienza University of Rome, Italy
}

\begin{abstract}
In this paper a non-hydrostatic and shock-capturing model for the simulation of wave-structure interaction and hydrodynamic phenomena (wave refraction, diffraction, shoaling and breaking) is proposed. The model is based on an integral formulation of the motion equations which are solved on a time dependent curvilinear coordinate system: a coordinate transformation maps the varying coordinates in the physical domain to a uniform transformed space. The motion equations are discretized by means of a shock-capturing numerical procedure based on high order WENO reconstructions. The solution procedure for the motion equations uses a five stage four order accurate Runge-Kutta (SSPRK) fractionalstep method and applies a pressure corrector formulation in order to obtain a divergence-free velocity field at each stage. The proposed model is validated against two benchmark test cases and is used in order to analyse the effects that a submerged breakwater produces on wave motion.

Keywords: non-hydrostatic model, $3 D$ simulation, high order shock-capturing WENO scheme, wave breaking, strong stability preserving Runge-Kutta (SSPRK), multigrid.
\end{abstract}

\section{Introduction}

The main problem to deal with in the study of nearshore hydrodynamics, is the wave transformation simulation from deep water to coastal regions. An efficient and well-tested tool for the simulation of the above mentioned phenomena is given by Boussinesq-type wave models with improved non linearity and dispersion characteristics (Gallerano et al. [1]). 
A different approach is based on the direct solution of the Navier-Stokes equations by using a tracking technique in order to follow the free surface elevation (maker-and-cell (MAC) and volume-of-fluid (VOF)). In many 3D models, Cartesian coordinates were used and thus the free surface normally crosses the computational cell arbitrarily. This leads to the difficulty of applying the pressure boundary condition precisely on the free surface and may eventually affect the accuracy of velocity computation nearby. An alternative approach can be obtained by assuming that the free surface is always placed at the upper computational boundary. Thus, the pressure boundary condition can be fixed precisely over the upper computational boundary. In order to solve the NavierStokes equations the pressure is decomposed in a hydrostatic and a nonhydrostatic component (Stelling and Zijlema [2], Yuan and $\mathrm{Wu}$ [3, 4], Young and Wu [5], Gallerano and Napoli [6], Gallerano and Cannata [7]). Stelling and Zijlema [2], proposed a model where the pressure is located at the cell faces rather than at the cell centres (known as the Keller-box method) in order to replace the staggered grids in the vertical direction. In this model, the pressure boundary conditions at the free surface are exactly assigned to zero without any approximation.

The integral form of the motion equations, expressed in terms of conserved variables, guarantees that high-order shock-capturing numerical schemes converge to correct weak solutions and are then able to directly simulate wave breaking and the energy dissipation associated with it (Toro [8], Gallerano and Cannata [9]). These schemes are front-capturing methods and are able to track the actual location of the wave breaking without requiring any criterion (Bradford [10]).

In this paper, the numerical simulation of wave transformation relies on the resolution of the motion equations expressed in an integral formulation on a time dependent curvilinear coordinate system: a coordinate transformation maps the varying coordinates in the physical domain to a uniform transformed space. The pressure boundary conditions are placed on the upper face of each computational cell. The solution procedure for motion equations uses a five stage four order accurate Runge-Kutta (SSPRK) fractional-step method and applies a pressure corrector formulation in order to obtain a divergence free velocity field at each stage.

In the prediction phase of the fractional-step proposed model the motion equations are discretized by means of a shock-capturing numerical procedure based on high order WENO reconstructions. The numerical flux is given at each cell interface by the solution of an exact Riemann problem. In the corrector phase of the fractional-step proposed model, in order to solve the Poisson equation and reduce the computational costs related to it, an alternate Zebra four-colour GaussSiedel relaxation method with a V-cycle multigrid strategy is used. In order to further reduce computational costs, the proposed model has been fully parallelized with Message Passing Interface (MPI). 


\section{Governing equations}

In order to express the integral formulation of the momentum equations in a time dependent coordinate system let us start from the intrinsic derivative of the momentum, that can be expressed as:

$$
\frac{D}{D t} \int_{\Delta V_{1}(t)} \rho u^{l} d V_{1}=\int_{\Delta V_{1}(t)} \frac{\partial \rho u^{l}}{\partial t} d V_{1}+\int_{\Delta A_{1}(t)} \rho u^{l} u^{m} n_{m} d A_{1}
$$

where $\Delta A_{1}(t)$ and $\Delta V_{1}(t)$ are, respectively, the surface and the volume of a material element of fluid that moves with velocity $u^{l}(l=1,3)$ defined in a Cartesian reference system of coordinates $x^{l}(l=1,3), n_{m}(m=1,3)$ is the outward unit normal vector to the surface $\Delta A_{1}(t)$ and $\rho$ is the density of the fluid. (The superscripts notation indicate components and not powers).

The three dimensional Leibniz integral rule can be written as:

$$
\frac{d}{d t} \int_{\Delta V_{2}(t)} \rho u^{l} d V_{2}=\int_{\Delta V_{2}(t)} \frac{\partial \rho u^{l}}{\partial t} d V_{2}+\int_{\Delta A_{2}(t)} \rho u^{l} v^{m} n_{m} d A_{2}
$$

where $\Delta V_{2}(t)$ is a time varying control volume that does not move with the fluid velocity, whose surface is given by $\Delta A_{2}(t)$ and where $v^{m}(m=1,3)$ is the velocity vector (defined in the Cartesian reference system) with which the $\Delta A_{2}(t)$ surface moves. (2):

It is assumed that at the instant $\Delta V_{1}(t)=\Delta V_{2}(t)=\Delta V(t)$. From eqns (1) and

$$
\frac{D}{D t} \int_{\Delta V(t)} \rho u^{l} d V=\frac{d}{d t} \int_{\Delta V(t)} \rho u^{l} d V+\int_{\Delta A(t)} \rho u^{l}\left(u^{m}-v^{m}\right) n_{m} d A
$$

By using the intrinsic derivative of the momentum (expressed by eqn. (3)) the integral form of the momentum equations in a time dependent coordinate system can be deduced; this equation is given by:

$$
\frac{\partial}{\partial t} \int_{\Delta V(t)} \rho u^{l} d V+\int_{\Delta A(t)} \rho u^{l}\left(u^{m}-v^{m}\right) n_{m} d A=\int_{\Delta V(t)} \rho f^{l} d V+\int_{\Delta A(t)} T^{l m} n_{m} d A
$$

where $f^{l}(l=1,3)$ represents the external body forces per unit mass vector and $T^{l m}$ the stress tensor.

The integral form of the continuity equation expressed in a time dependent coordinate system is given by:

$$
\frac{d}{d \tau} \int_{\Delta V(\tau)} \rho d V+\int_{\Delta A(\tau)} \rho\left(u^{m}-v^{m}\right) n_{m} d A=0 .
$$

In order to simulate the fully dispersive wave processes eqn (4) can be transformed in the following way. Let $H\left(x^{1}, x^{2}, t\right)=h\left(x^{1}, x^{2}, t\right)+\eta\left(x^{1}, x^{2}, t\right)$ 
where $h$ is the water depth and $\eta$ is the surface elevation. In order to accurately represent the bottom and surface geometry we consider a transformation from the Cartesian reference system of coordinates $\left(x^{1}, x^{2}, x^{3}, t\right)$ to the curvilinear reference system of coordinates $\left(\xi^{1}, \xi^{2}, \xi^{3}, \tau\right)$ where:

$$
\xi^{1}=x^{1}, \quad \xi^{2}=x^{2}, \quad \xi^{3}=\frac{x^{3}+h}{H}, \quad \tau=t
$$

the following relation is valid:

$$
\frac{\partial \xi^{3}}{\partial \tau}=-\frac{\xi^{3}}{H} \frac{\partial H}{\partial \tau}
$$

This coordinate transformation basically maps the varying vertical coordinates in the physical domain to a uniform transformed space where $\xi^{3}$ spans from 0 to 1.

We define the transformation matrix $C_{m}^{l}=\partial x^{l} / \partial \xi^{m}$ and its inverse $\bar{C}_{m}^{l}=$ $\partial \xi^{l} / \partial x^{m}(l, m=1,3)$. The metric tensor and its inverse are defined by $g_{l m}=$ $C_{l}^{k} C_{m}^{k}$ and $g^{l m}=\bar{C}_{l}^{k} \bar{C}_{m}^{k}$, respectively. The Jacobian of the transformation is defined by $\sqrt{g}=\operatorname{det}\left(C_{m}^{l}\right)$. It is not difficult to verify that in the particular case of the above-mentioned transformation $\sqrt{g}=H$.

For an incompressible fluid, eqn. (4) expressed in the time dependent coordinate system $\left(\xi^{1}, \xi^{2}, \xi^{3}, \tau\right)$, transformed from the Cartesian reference system of coordinates $\left(x^{1}, x^{2}, x^{3}, t\right)$ by eqn (6), and with the external body forces given by the only gravitational force, becomes:

$$
\begin{aligned}
& \quad \frac{\partial}{\partial \tau} \int_{\Delta V(\tau)} u^{l} d V+\int_{\Delta A(\tau)} u^{l}\left(u^{m}-v^{m}\right) n_{m} d A=-\int_{\Delta V(\tau)} G(H-h)_{, l} d V \\
& -\int_{\Delta V(\tau)} q_{, l} d V+\int_{\Delta A(\tau)} \frac{T^{l m}}{\rho} n_{m} d A
\end{aligned}
$$

where $G$ is the constant of gravity and the comma with an index in subscript denotes the derivative as []$_{, l}=\partial[] / \partial x^{l}$. The time dependent coordinate system moves with velocity given by $v^{m}=\left\{0,0,-\frac{\xi^{3}}{H} \frac{\partial H}{\partial \tau}\right\}$. The first integral on the right hand side of eqn. (8) is related to the hydrostatic pressure gradient; the second integral on the right hand side is related to the dynamic pressure gradient.

Let us introduce a restrictive condition on the volume element $\Delta V$ : in the following, $\Delta V$ must be considered as a volume element that is bounded by curves lying on the coordinate lines. Recalling $\sqrt{g}=H, d V=\sqrt{g} d \xi^{1} d \xi^{2} d \xi^{3}$ and defining with:

$$
\begin{gathered}
\Delta A_{x y}=\Delta \xi^{1} \Delta \xi^{2}, \quad \Delta V=\Delta \xi^{1} \Delta \xi^{2} \Delta \xi^{3}, \quad \bar{H}=\frac{1}{\Delta V(\tau)} \int_{\Delta V(\tau)} H d \xi^{1} d \xi^{2} d \xi^{3} \\
\bar{u}^{l}=\frac{1}{\Delta V(\tau)} \int_{\Delta V(\tau)} u^{l} d \xi^{1} d \xi^{2} d \xi^{3}, \quad \overline{H u^{l}}=\frac{1}{\Delta V(\tau)} \int_{\Delta V(\tau)} u{ }^{l} H d \xi^{1} d \xi^{2} d \xi^{3},
\end{gathered}
$$


eqn (8) is transformed in:

$$
\begin{gathered}
\frac{\partial \overline{H u^{l}}}{\partial \tau}=-\frac{1}{\Delta V(\tau)} \sum_{\alpha=1}^{3}\left\{\int_{\Delta A^{\alpha+}}\left[H u^{l}\left(u^{\alpha}-v^{\alpha}\right)+\frac{1}{2} G H^{2}\right] d \xi^{\beta} d \xi^{\gamma}-\int_{\Delta A^{\alpha-}}\left[H u^{l}\left(u^{\alpha}-v^{\alpha}\right)\right.\right. \\
\left.\left.+\frac{1}{2} G H^{2}\right] d \xi^{\beta} d \xi^{\gamma}\right\}+\frac{1}{\Delta V(\tau)} \sum_{\alpha=1}^{3}\left\{\int_{\Delta A^{\alpha+}} G h H d \xi^{\beta} d \xi^{\gamma}-\int_{\Delta A^{\alpha-}} G h H d \xi^{\beta} d \xi^{\gamma}\right\} \\
-\frac{1}{\Delta V(\tau)} \int_{\Delta V(\tau)} q_{, l} H d \xi^{1} d \xi^{2} d \xi^{3}+\frac{1}{\Delta V(\tau)} \sum_{\alpha=1}^{3}\left\{\int_{\Delta A^{\alpha+}} \frac{H T^{l \alpha}}{\rho} d \xi^{\beta} d \xi^{\gamma}-\int_{\Delta A^{\alpha-}} \frac{H T^{l \alpha}}{\rho} d \xi^{\beta} d \xi^{\gamma}\right\}
\end{gathered}
$$

where $\Delta A^{\alpha+}$ and $\Delta A^{\alpha-}$ indicate the contour surfaces of the volume element $\Delta V$ on which $\xi^{\alpha}$ is constant and which are located at the larger and at the smaller value of $\xi^{\alpha}$ respectively. Here the indexes $\alpha, \beta$ and $\gamma$ are ciclic.

Let us integrate the continuity equation (5) over the particular volume element $\Delta \tilde{V}=\Delta A_{x y} \bar{H}$ :

$$
\frac{\partial \bar{H}}{\partial \tau}=\frac{1}{\Delta A_{x y}} \int_{0}^{1} \sum_{\alpha=1}^{2}\left[\int_{\Delta A^{\alpha+}} H u^{\alpha} d \xi^{\beta}-\int_{\Delta A^{\alpha-}} H u^{\alpha} d \xi^{\beta}\right]=0 .
$$

Eqn. (11) represents the governing equation for the surface movements. Eqns (10) and (11) represent the expressions of the three-dimensional motion equations as a function of the $\overline{H u^{l}}$ and $\bar{H}$ variables in the time dependent coordinate system $\left(\xi^{1}, \xi^{2}, \xi^{3}, \tau\right)$. The numerical integration of the above mentioned eqns (10) and (11) allows the fully dispersive wave processes simulation.

The state of the system is known at the centre of the calculation cells and it is defined by the cell-averaged values of $\overline{H u^{l}}$ and $\bar{H} \cdot \tau^{(n)}$ is the time level of the known variables while $\tau^{(n+1)}$ is the time level of the unknown variables. The solution procedure uses a five stage fourth order accurate Strong Stability Preserving Runge-Kutta (SSPRK) fractional-step method for eqn (10) and applies a pressure correction formulation to obtain a divergence free velocity field at each time level. With ${\overline{H u^{l}}}^{(n)}$ known, ${\overline{H u^{l}}}^{(n+1)}$ is calculated with the following five stage iteration procedure. Let:

$$
{\overline{H u^{l}}}^{(0)}={\overline{H u^{l}}}^{(n)} \text {. }
$$

At each stage $p$ (ove $p=1,2, \ldots, 5$ ) an auxiliary field ${\overline{H u^{l}}}_{*}^{(p)}$ is obtained directly from eqn. (10) using values from the previous stage:

$$
{\overline{H u^{l}}}_{*}^{(p)}=\sum_{q=0}^{p-1}\left\{\Omega_{p q}{\overline{H u^{l}}}^{(q)}+\Delta \tau \varphi_{p q} D\left[H u^{l(q)}, \tau^{(n)}+d_{q} \Delta \tau\right]\right\}
$$

where $D\left(H, u^{l}, \tau\right)$ indicates the right hand side of eqn. (10), in which the term related to the dynamic pressure gradient is omitted. See Spiteri and Ruuth [11] for the values of coefficients $\Omega_{p q}, \varphi_{p q}$ and $d_{q}$. The auxiliary velocity field $\bar{u}_{*}^{l}(p)$ (associated to the auxiliary variable $\overline{H u}^{(}{ }_{*}$ ) calculated by eqn. (13)) will not satisfy the continuity equation. As a result, the velocity and the pressure fields are 
corrected, at each intermediate step $p$, by introducing a scalar potential $\Psi$ which is calculated by the well-known Poisson pressure equation that in integral form is given by:

$$
\int_{\Delta A(\tau)} \frac{\partial \Psi^{(p)}}{\partial x_{l}} n_{l} d A=-\int_{\Delta A(\tau)} \bar{u}_{*}^{(p)} n_{l} d A
$$

The corrector irrotational velocity field is calculated by the following expression:

$$
\bar{u}_{c}^{(p)}=\frac{\partial \Psi^{(p)}}{\partial x_{l}},
$$

from which:

$$
\bar{u}^{l(p)}=\bar{u}_{*}^{l(p)}+\bar{u}_{c}^{l(p)} .
$$

Let us indicate with $L\left(H, u^{l}, \tau\right)$ the right hand side of eqn (11). The advancing at the $p$ stage of the depth $\bar{H}^{(p)}$ is obtained by:

$$
\bar{H}^{(p)}=\bar{H}^{(p-1)}+L\left(H^{(p-1)}, u^{l(p-1)}, \tau^{n}+\Delta \tau\right) .
$$

The value of ${\overline{H u^{l}}}^{(n+1)}$ is given by:

$$
{\overline{H u^{l}}}^{(n+1)}={\overline{H u^{l}}}^{(5)} \text {. }
$$

For the calculation of term $D\left(H, u^{l}, \tau\right)$ e $L\left(H, u^{l}, \tau\right)$ the numerical approximations of integrals on the right hand side of eqns (10) and (11) is required. The aforementioned calculation is based on the following sequence.

1. High order WENO reconstructions, from cell averaged values, of the point values of the unknown variables at the centre of the contour face which define the calculation cells. At the centre of the contour face which is common with two adjacent cells, two point values of the unknown variables are reconstructed by means of two WENO reconstruction defined on two adjacent cells.

2. Advancing in time of the point values of the unknown variables at the centre of the contour face by means of the so-called exact solution of the Riemann problem, with initial data given by the pair of point-values computed by two WENO reconstructions defined on the two adjacent cells.

3. Calculation of the spatial integrals which define $D\left(H, u^{l}, \tau\right)$ e $L\left(H, u^{l}, \tau\right)$. For a deep insight into the WENO reconstructions, into the advancing in time of the unknown variables and into the calculation of the spatial integrals which define $D\left(H, u^{l}, \tau\right)$ and $L\left(H, u^{l}, \tau\right)$ see Gallerano et al. [12]. 


\section{Results}

The proposed model has been validated against two benchmark test cases. First, a validation test based on the laboratory experiment proposed by Beji and Battjes [13] is shown, in order to verify the model ability to reproduce wave transformation over a submerged breakwater. Furthermore, a validation test based on the laboratory experiment proposed by Berkhoff [14] is shown, in order to verify the model ability to reproduce wave deformation (refraction and diffraction) due to a shoal on a uniform sloped bottom. Finally, the model is used in order to numerically simulate wave propagation over a submerged breakwater and the wet and dry front in the swash zone.

\subsection{Periodic wave over a submerged bar}

The first test case simulates wave propagation over a submerged breakwater. In particular, it is possible to observe how the proposed model succeeds in the simulation of the wave shoaling. The proposed non-hydrostatic model is validated using the experimental data produced by Beji and Battjes [13]. The experimental channel has a length of $30 \mathrm{~m}$ and the still water depth is $0.4 \mathrm{~m}$ which is reduced to $0.1 \mathrm{~m}$ over the submerged breakwater. The offshore slope of the breakwater is $1 / 20$ and the onshore slope is $1 / 10$. A wave train characterized by a period of $2.02 \mathrm{~s}$ and amplitude $1.0 \mathrm{~cm}$ is incident from the left boundary of the channel. Fig. 1 shows, over six different sections, the comparison between numerical results and experimental data. Fig. 1(a), for section $x=10.5 \mathrm{~m}$, and fig. 1(b), for section $x=12.5 \mathrm{~m}$, show that the proposed model respectively reproduce wave shoaling and wave propagation over the breakwater with good agreement with the experimental data.

\subsection{Wave deformation by an elliptic shoal on a sloped bottom}

An experiment extracted from "Report W. 154-VIII" of the Delft Hydraulics Laboratory, by Berkhoff [14], is numerically reproduced. This experiment is carried out in order to verify the ability of the proposed hydrodynamic model to simulate physical process of wave propagation and wave deformation (refraction and diffraction) due to a shoal.

The comparison between the numerical results and the measured data from Berkhoff [14] is shown. Berkhoff [14] carried out a laboratory study of monochromatic wave propagation over an elliptic shoal located on a plane slope of $1 / 50$.

In fig. 2(a) and 2(b), the comparison between the numerical results and the measured data of the wave height relative to section $x=2 \mathrm{~m}$ and $x=0 \mathrm{~m}$ are respectively shown. The computed wave height is obtained by taking the difference between maximum and minimum free-surface elevation considered over a time interval in which the wave form is permanent.

From the figures it can be seen that the model results are close to the experimental data. In particular, from fig. 2(a) it can be observed that there is a slightly over prediction of the maximum value of the wave height. 


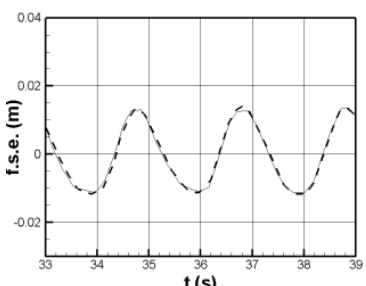

(a)

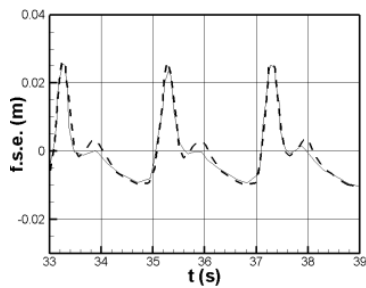

(c)

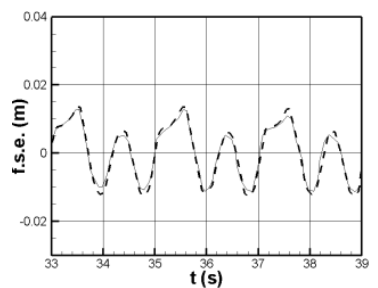

(e)

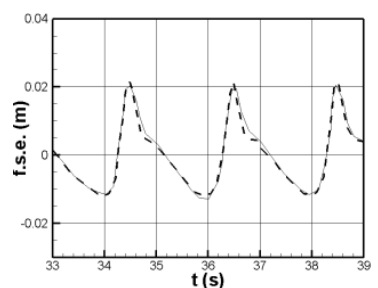

(b)

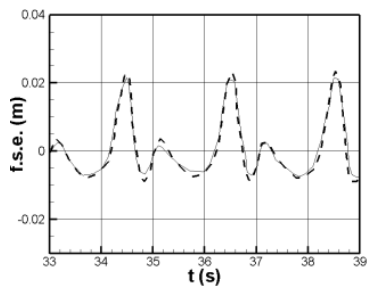

(d)

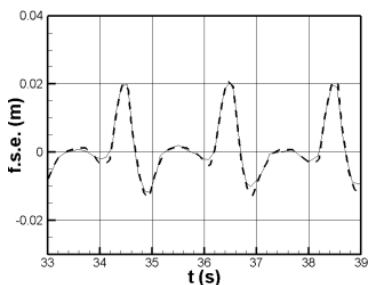

(f)

Figure 1: Wave over a submerged bar. Comparison between numerical results (solid line) and experimental data (dashed line) of the surface elevation at (a) $x=10.5 \mathrm{~m}$; (b) $x=12.5 \mathrm{~m}$; (c) $x=13.5 \mathrm{~m}$; (d) $x=14.5 \mathrm{~m}$; (e) $x=15.7 \mathrm{~m}$; (f) $x=17.3 \mathrm{~m}$.

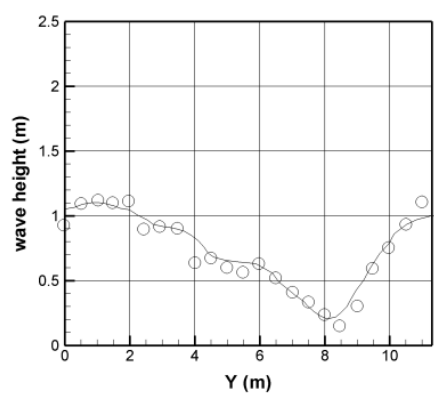

(a)

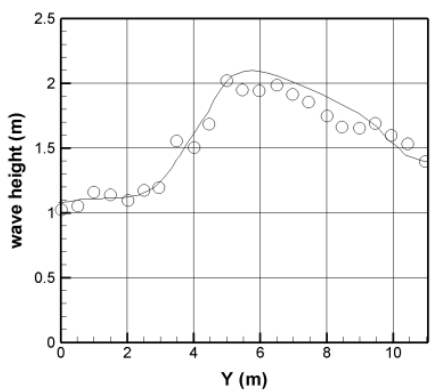

(b)

Figure 2: Wave over an elliptic shoal. Comparison between numerical results (solid line) and experimental data (circles) of the wave heights along sections (a) $x=2 \mathrm{~m}$ and (b) $x=0 \mathrm{~m}$. 


\subsection{Wave propagation over a submerged breakwater}

The effectiveness of the proposed model in reproducing the effects of wavecoastal structures interactions and simulating the propagation of the wet and dry front is shown.

The numerical simulation reproduces a single wave propagation over a plane beach where a submerged breakwater is located. The wave period is $3 \mathrm{~s}$ and the wave amplitude is $0.1 \mathrm{~m}$. The computational domain is $40 \mathrm{~m}$ long and the beach has a 0.008 slope. The still water depth in the generation zone is about $1.6 \mathrm{~m}$. The spatial discretization steps are $\Delta x=0.075 \mathrm{~m}$ and $\Delta z=0.09 \mathrm{~m}$ while the time discretization step is $\Delta t=0.001 \mathrm{~s}$. In fig. 3 the free surface elevation over the submerged breakwater is shown underlining the ability of the proposed model to catch the structure effects of the hydrodynamic field. In particular, it is possible to observe how the presence of the submerged breakwater modifies the wave profile and substantially reduces the downstream current velocity.

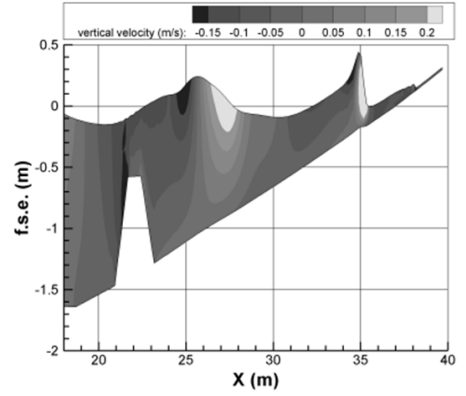

(a)

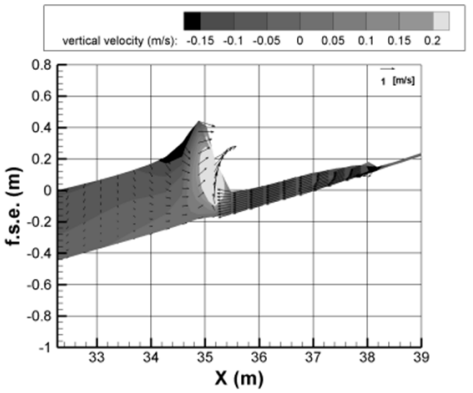

(b)

Figure 3: Wave over a submerged breakwater. (a) Free surface elevation. (b) Free surface elevation detail and velocity field (black vectors) in the breaking zone and in the surf zone (only one vector out of two is represented).

\section{Conclusion}

In this paper a non-hydrostatic and shock-capturing model for the simulation of hydrodynamic phenomena (wave refraction, diffraction, shoaling and breaking) and wave-structure interaction is proposed. The model is based on an integral formulation of the motion equations that are solved on a time dependent curvilinear coordinate system: a coordinate transformation maps the varying coordinates in the physical domain to a uniform transformed space in order to accurately take into account the bed and the free surface elevation profile. The comparison between the numerical results and the experimental data shows that the proposed model based on the transformation from the physical to the uniform transformed space and the high order WENO shock-capturing procedure is able to simulate the effects that a submerged breakwater produces on wave motion. 


\section{References}

[1] Gallerano, F., Cannata, G. \& Villani, M., An integral contravariant formulation of the fully non-linear Boussinesq equations. Coastal Engineering, 136, pp. 83-119, 2014.

[2] Stelling, G. \& Zijlema, M., An accurate and efficient finite-difference algorithm for non-hydrostatic free-surface flow with application to wave propagation. Int. J. Numer. Methods Fluids, 43, pp. 1-23, 2003.

[3] Yuan, H. \& Wu, C.-H., A two-dimensional vertical non-hydrostatic sigma model with an implicit method for free-surface flows. Int. J. Numer. Methods Fluids, 44, pp. 811-835, 2004a.

[4] Yuan, H. \& Wu, C.-H., An implicit three-dimensional fully non-hydrostatic model for free-surface flows. Int. J. Numer. Methods Fluids, 46, pp. 709733, 2004b.

[5] Young, C.-C. \& Wu, C.-H., A $\sigma$-coordinate non-hydrostatic model with embedded Boussinesq-type-like equations for modeling deep-water waves. Int. J. Numer. Methods Fluids, 63, pp. 1448-1470, 2010.

[6] Gallerano, F., Napoli, E., A dynamic subgrid-scale tensorial eddy viscosity model. Continuum Mechanics and Thermodynamics, 11(1), pp. 1-14, 1999.

[7] Gallerano, F., Cannata, G., Compatibility of reservoir sediment flushing and river protection. Journal of Hydraulic Engineering, 137(10), pp. 11111125, 2011.

[8] Toro, E.F., Riemann Solvers and Numerical Methods for Fluid Dynamics. Springer, 2009.

[9] Gallerano, F., Cannata, G., Central WENO scheme for the integral form of contravariant shallow-water equations. International Journal for Numerical Methods in Fluids, 67(8), pp. 939-959, 2011.

[10] Bradford, S.F., Godunov-based model for non-hydrostatic wave dynamics. J. Waterway Port Coastal Ocean Eng., 131, pp. 226-238, 2005.

[11] Spiteri, R.J. \& Ruuth, S.J., A new class of optimal high-order strongstability- preserving time discretization methods. SIAM J Numer Anal, 40(2), pp. 469-491, 2002.

[12] Gallerano, F., Cannata, G. \& Tamburrino, M., Upwind WENO scheme for Shallow Water Equations in contravariant formulation. Computers \& Fluids, 62, pp. 1-12, 2012.

[13] Beji, S. \& Battjes, J.A., Experimental investigation of wave propagation over a bar. Coastal Eng, 19, pp. 151-162, 1993.

[14] Berkhoff, J.C.W., Refraction and diffraction of water waves: wave deformation by a shoal. Report W. 154-VIII, Delft Hydraulics Laboratory, 1982. 